Open Access

\title{
Why do patients get idiopathic pulmonary fibrosis? Current concepts in the pathogenesis of pulmonary fibrosis
}

Pierre-Simon Bellaye $e^{1,2}$ and Martin Kolb $b^{1,2^{*}}$

\begin{abstract}
Idiopathic pulmonary fibrosis (IPF) is a devastating lung disease of unknown origin. Recent findings suggest that IPF results from multiple factors that eventually lead to interstitial lung injury. In the pathogenesis it is likely that complex relationships between genetic predispositions, environmental exposures, and lung infections promote the fibrotic processes causing IPF; it is this complexity and the multiplicity of causes that make the population and clinical course of IPF so heterogeneous. Thus, it is clear that one common factor driving IPF pathogenesis in all patients would be far too simplified of an understanding. In recent years, efforts have been made in finding therapeutic strategies that target disease progression rather than disease onset. The biochemical composition and abnormal stiffness of the matrix might be crucial in controlling the cellular phenotype in fibrotic lungs that promotes disease progression and persistence. Though there has been substantial progress in the IPF field in recent years, much more work is required in order to improve the prognosis associated with this disease.
\end{abstract}

Keywords: Extracellular matrix, Fibrogenesis, Idiopathic pulmonary fibrosis, Lung injury, Myofibroblast, Wound healing

\section{Background}

Idiopathic pulmonary fibrosis (IPF) is one of the most common diseases classified as a rare disease. The prognosis of IPF is poor, with most patients succumbing to their illness at a rate comparable to aggressive cancers [1]. The causes of IPF remain elusive and are not easy to identify as patients diagnosed are typically at an advanced stage of the disease. Several associated risk factors without a clear causative role have been reported [2], including environmental and occupational exposures, infections, and genetic polymorphisms. To date, there is no ideal therapy for IPF, but at least two drugs have been approved in recent years, both demonstrating a significant impact on disease progression [3, 4]. Understanding the signals involved in the pathogenesis and progression of IPF remain a critical component in discovering new therapies, providing early diagnosis, and preventing disease progression.

\footnotetext{
* Correspondence: kolbm@mcmaster.ca

${ }^{1}$ Firestone Institute for Respiratory Health, St. Joseph's Healthcare and Department of Medicine, McMaster University Hamilton, Hamilton, Canada ${ }^{2}$ Department of Pathology and Molecular Medicine, McMaster University Hamilton, Hamilton, Canada
}

Is IPF an uncontrolled protective process?

Unlike fish or amphibians, evolution has favoured fibrogenesis over regeneration in complex organisms such as mammals $[5,6]$. Even if the selective advantage provided by such a "substitution" remains unclear, fibrogenesis certainly benefits survival by preventing blood loss and pathogen invasion through mechanisms of wound closure. The pathological evolution of fibrosis requires the combination of an initial injury, which starts the healing process, and impaired wound healing mechanisms. This view suggests an interaction between environmental and genetic factors in IPF pathogenesis. Several gene mutations have been identified among IPF patients in recent years [7], but whether they are direct cause, predisposition factors, or just associations remains unclear. For instance, surfactant protein and mucin gene mutations can lead to direct epithelial cell injury and death, whereas telomerase gene mutations predispose the epithelium to a pathologic response by favouring an abnormal turnover and repair. However, these mutations only affect $1 \%$ (surfactant), $35 \%$ (mucins), and $3 \%$ (telomeres) of IPF patients, leaving more the $60 \%$ of patients without identified genetic predispositions [8]. Therefore, IPF 
pathogenesis has to be seen as a process involving several steps in which genetic mutations could represent just one of many important components.

Exposure to inhaled environmental agents, most of all cigarette smoke, represents an important risk factor for IPF. The increased risk of developing IPF remains even after smoke cessation, suggesting the establishment of self-sustained (or autocrine) mechanisms after the initial injury $[9,10]$. Cigarette smoke, in addition to epithelial injury, also influences epigenetic changes such as DNA methylation and chromatin modifications that regulate the expression of genes involved in tissue repair and which have an impact on IPF pathogenesis [7]. Infections are common in the IPF population; numerous viruses and bacteria have the potential to cause epithelial cell injury and apoptosis [11] and have the capacity to modulate the host response to injury. In experimental settings, infections seem only able to worsen fibrosis in conjugation with other profibrotic stimuli, suggesting that infections might be co-factors for IPF $[12,13]$. In the past decade, the lack of clinical evidence of an ongoing inflammation, as well as the inefficiency of immunosuppressive therapies in IPF, diminished the role of chronic inflammation in IPF pathogenesis [5]. Nevertheless, it cannot be ignored that inflammatory cytokine and immune cell infiltration are found in IPF $[14,15]$. We have shown, in vivo, that interleukin- $1 \beta$ induces an early inflammation promoting the activation of profibrotic pathways through transforming growth factor (TGF)- $\beta 1$, able to self-sustain up to day 60 independently of any signs of residual inflammation and trigger clustering of myofibroblasts and collagen similar to myofibroblastic foci observed in humans [16]. This highlights complex relations between the initial injury and the impaired wound healing that might favour the profibrotic processes which lead to IPF.

\section{The vicious cycle caused by increased lung stiffness}

Myofibroblasts are the major producers of the fibrotic extracellular matrix (ECM) which results in the characteristic stiffness of a fibrotic lung, decreased lung volumes, and shortness of breath in patients. In vitro, the differentiation of myofibroblasts is strongly correlated with substrate stiffness; it is clear that stiff substrates promote the production of profibrotic mediators and ECM deposition [17, 18], whereas substrates of physiological stiffness inactivate myofibroblasts and favour apoptosis [19]. Activation of Rho Kinase and Focal Adhesion Kinase by increased force tension appears to have a major role in this process and the inhibition of these pathways prevents experimental fibrosis [20-22]. The ECM is a storage of growth factors, such as latent TGF- $\beta 1$, which are bound to integrins, transmembrane proteins allowing cell-matrix adhesions. An increase in substrate stiffness induces mechanical resistance that favours the release of active TGF- $\beta 1$ from the integrin promoting myofibroblast activity [23]. These in vitro approaches strongly suggest that stiffness alone can drive myofibroblast activation and subsequent ECM deposition. However, they lack the spatial cues that ECM and growth factors encounter in the $3 \mathrm{D}$ fibrotic lung in vivo. Booth et al. [24] showed, very elegantly, that decellularised matrix from IPF but not healthy lungs can drive myofibroblast differentiation and accumulation. Even though the difference in stiffness between non-IPF and IPF lungs was conserved after decellularisation in these studies, it remains unclear whether the altered stiffness alone is responsible for the difference of cell behaviour between the two types of matrix or whether the abnormal composition of the fibrotic ECM also plays a role. Indeed, the "matrisome" of the IPF lung is completely different from the non-IPF lung with many more ECM components as well as more latent TGF- $\beta 1$ trapped in the matrix [24]. These new techniques are very useful to mimic the native environment of cells, but also have limitations including heterogeneous and non-physiological stiffness of the acellular matrix [25]. Moreover, no study has yet fully described the effect of decellularisation on ECM component preservation and it is possible that proteins trapped in the matrix, which may have a role on cell behaviour, are washed out through the process. For instance, Parker et al. [26] demonstrated that the IPF matrix can drive the expression of genes in fibroblasts already highly present in the diseased ECM. This suggests an autocrine feedback loop in which IPF ECM triggers the upregulation of its own abnormal ECM components. This shows that the biochemical composition could be as important as the stiffness of the matrix in controlling the cellular phenotype in fibrotic lungs [26]. Further studies are needed to elucidate the actual contribution of matrix stiffness and composition on myofibroblast differentiation and persistence.

\section{Conclusions}

IPF is a complex disease involving multiple steps which eventually overcome physiological repair mechanisms and lead to fibrosis. Even if the etiologic events causing the onset of IPF remain unknown, decades of research have highlighted the fact that fibrogenesis requires a combination of several factors which cause both epithelial injury and impaired wound healing. It is this complexity and the multiplicity of causes that make the population and clinical course of IPF so heterogeneous. For the time being, it seems more realistic to continue investigating therapeutic strategies that limit disease progression rather that prevent its development. Due to the multiple pathways involved in abnormal fibrogenesis, multi-target therapies appear essential. 


\section{Abbreviations}

ECM: Extracellular matrix; IPF: Idiopathic pulmonary fibrosis; TGF- $\beta 1$ : Transforming growth factor- $\beta 1$

\section{Competing interests}

The authors declare that they have no competing interests.

\section{Authors' information}

Martin Kolb is professor of the Division of Respirology at McMaster University and the Research Director at the Firestone Institute for Respiratory Health (FIRH) at Hamilton, ON, Canada. Pierre-Simon Bellaye is a post-doctoral fellow at the Division of Respirology at McMaster University. Their research focuses on the role of abnormal extracellular matrix properties on IPF progression.

\section{Acknowledgements}

The authors would like to thank Philipp Kolb for his help with the writing of the manuscript. Pierre-Simon Bellaye is funded by le Fonds de Dotation "Recherche en Santé Respiratoire" et de la Fondation du Souffle and the Canadian Pulmonary Fibrosis Foundation.

Received: 24 June 2015 Accepted: 25 June 2015

Published online: 24 September 2015

\section{References}

1. Vancheri C, Failla M, Crimi N, Raghu G. Idiopathic pulmonary fibrosis: a disease with similarities and links to cancer biology. Eur Respir J. 2010;35:496-504.

2. Ley B, Collard HR. Epidemiology of idiopathic pulmonary fibrosis. Clin Epidemiol. 2013;5:483-92.

3. Noble PW, Albera C, Bradford WZ, Costabel U, Glassberg MK, Kardatzke D, et al. Pirfenidone in patients with idiopathic pulmonary fibrosis (CAPACITY) two randomised trials. Lancet. 2011;377:1760-9.

4. Mazzei ME, Richeldi L, Collard HR. Nintedanib in the treatment of idiopathic pulmonary fibrosis. Ther Adv Respir Dis. 2015;9:121-9.

5. Thannickal VJ, Zhou Y, Gaggar A, Duncan SR. Fibrosis: ultimate and proximate causes. J Clin Invest. 2014;124:4673-7.

6. Gurtner GC, Werner S, Barrandon Y, Longaker MT. Wound repair and regeneration. Nature. 2008;453:314-21.

7. Yang IV, Schwartz DA. Epigenetics of idiopathic pulmonary fibrosis. Biochem Cell Biol. 2015;165:48-60.

8. Wolters PJ, Collard HR, Jones KD. Pathogenesis of idiopathic pulmonary fibrosis. Annu Rev Pathol. 2014;9:157-79.

9. Samara KD, Margaritopoulos G, Wells AU, Siafakas NM, Antoniou KM Smoking and pulmonary fibrosis: novel insights. Pulm Med. 2011;2011:461439.

10. Spira A, Beane J, Shah V, Liu G, Schembri F, Yang X, et al. Effects of cigarette smoke on the human airway epithelial cell transcriptome. Proc Natl Acad Sci U S A. 2004;101:10143-8.

11. Isler JA, Skalet AH, Alwine JC. Human cytomegalovirus infection activates and regulates the unfolded protein response. J Virol. 2005;79:6890-9.

12. Lok SS, Haider Y, Howell D, Stewart JP, Hasleton PS, Egan JJ. Murine gammaherpes virus as a cofactor in the development of pulmonary fibrosis in bleomycin resistant mice. Eur Respir J. 2002;20:1228-32.

13. Knippenberg S, Ueberberg B, Maus R, Bohling J, Ding N, Tort Tarres M, et al. Streptococcus pneumoniae triggers progression of pulmonary fibrosis through pneumolysin. Thorax. 2015;70:636-46.

14. Boomars KA, Schweizer RC, Zanen P, van den Bosch JM, Lammers JW, Koenderman L. Eosinophil chemotactic activity in bronchoalveolar lavage from idiopathic pulmonary fibrosis is dependent on cytokine priming of eosinophils. Eur Respir J. 1998;11:1009-14.

15. Daniil Z, Kitsanta P, Kapotsis G, Mathioudaki M, Kollintza A, Karatza M, et al. CD8+ T lymphocytes in lung tissue from patients with idiopathic pulmonary fibrosis. Respir Res. 2005;6:81

16. Kolb M, Margetts PJ, Anthony DC, Pitossi F, Gauldie J. Transient expression of IL-1 beta induces acute lung injury and chronic repair leading to pulmonary fibrosis. J Clin Invest. 2001;107:1529-36.

17. Eisenberg JL, Safi A, Wei X, Espinosa HD, Budinger GS, Takawira D, et al. Substrate stiffness regulates extracellular matrix deposition by alveolar epithelial cells. Res Rep Biol. 2011;2011:1-12.

18. Balestrini JL, Chaudhry S, Sarrazy V, Koehler A, Hinz B. The mechanical memory of lung myofibroblasts. Integr Biol (Camb). 2012;4:410-21.
19. Marinkovic A, Liu F, Tschumperlin DJ. Matrices of physiologic stiffness potently inactivate idiopathic pulmonary fibrosis fibroblasts. Am J Respir Cell Mol Biol. 2013;48:422-30.

20. Huang X, Yang N, Fiore VF, Barker TH, Sun Y, Morris SW, et al. Matrix stiffness-induced myofibroblast differentiation is mediated by intrinsic mechanotransduction. Am J Respir Cell Mol Biol. 2012;47:340-8.

21. Zhou Y, Huang X, Hecker L, Kurundkar D, Kurundkar A, Liu H, et al. Inhibition of mechanosensitive signaling in myofibroblasts ameliorates experimental pulmonary fibrosis. J Clin Invest. 2013;123:1096-108.

22. Lagares D, Busnadiego O, Garcia-Fernandez RA, Kapoor M, Liu S, Carter DE, et al. Inhibition of focal adhesion kinase prevents experimental lung fibrosis and myofibroblast formation. Arthritis Rheum. 2012;64:1653-64.

23. Wipff PJ, Hinz B. Integrins and the activation of latent transforming growth factor beta1 - an intimate relationship. Eur J Cell Biol. 2008;87:601-15.

24. Booth AJ, Hadley R, Cornett AM, Dreffs AA, Matthes SA, Tsui JL, et al. Acellular normal and fibrotic human lung matrices as a culture system for in vitro investigation. Am J Respir Crit Care Med. 2012;186:866-76.

25. Melo E, Garreta E, Luque T, Cortiella J, Nichols J, Navajas D, et al. Effects of the decellularization method on the local stiffness of acellular lungs. Tissue Eng Part C Methods. 2014;20:412-22.

26. Parker MW, Rossi D, Peterson M, Smith K, Sikstrom K, White ES, et al. Fibrotic extracellular matrix activates a profibrotic positive feedback loop. J Clin Invest. 2014;124:1622-35

\section{Submit your next manuscript to BioMed Central and take full advantage of:}

- Convenient online submission

- Thorough peer review

- No space constraints or color figure charges

- Immediate publication on acceptance

- Inclusion in PubMed, CAS, Scopus and Google Scholar

- Research which is freely available for redistribution 